\title{
Hepatic and Neuromuscular Forms of Glycogen Storage Disease Type IV Caused by Mutations in the Same Glycogen-branching Enzyme Gene
}

\author{
Yong Bao, Priya Kishnani, Jer-Yuarn Wu, and Yuan-Tsong Chen \\ Department of Pediatrics, Duke University Medical Center, Durham, North Carolina 27710
}

\begin{abstract}
Glycogen storage disease type IV (GSD-IV) is an autosomal recessive disease resulting from deficient glycogen-branching enzyme (GBE) activity. The classic and most common form is progressive liver cirrhosis and failure leading to either liver transplantation or death by $5 \mathrm{yr}$ of age. However, the liver disease is not always progressive. In addition, a neuromuscular type of the disease has been reported. The molecular basis of GSD-IV is not known, nor is there a known reason for the clinical variability. We studied the GBE gene in patients with various presentations of GSDIV. Three point mutations in the GBE gene were found in two patients with the classical presentation: R515C, F257L, and R524X. Transient expression experiments showed that these mutations inactivated GBE activity. Two point mutations, L224P and Y329S, were detected in two separate alleles of a patient with the nonprogressive hepatic form. The L224P resulted in complete loss of GBE activity, whereas the Y329S resulted in loss of $\sim 50 \%$ of GBE activity. The Y329S allele was also detected in another patient with the nonprogressive form of GSD-IV but not in 35 unrelated controls or in patients with the more severe forms of GSDIV. A 210-bp deletion from nucleotide 873 to 1082 of the GBE cDNA was detected in a patient with the fatal neonatal neuromuscular presentation. This deletion, representing the loss of one full exon, was caused by a $3^{\prime}$ acceptor splicing site mutation ( $a g$ to $a$ a). The deletion abolished GBE activity. Our studies indicate that the three different forms of GSD-IV were caused by mutations in the same GBE gene. The data also suggest that the significant retention of GBE activity in the Y329S allele may be a reason for the mild disease. Further study of genotype/phenotype correlations may yield useful information in predicting the clinical outcomes. (J. Clin. Invest. 1996. 97:941-948.) Key words: human glycogen-branching enzyme - type IV glycogen storage disease - mutation analysis • inborn error of metabolism
\end{abstract}

Portions of this work were presented at the annual meeting of the American Society of Human Genetics in Montréal, Canada, on 24-28 October 1994.

Address correspondence to Y.-T. Chen, M.D., Ph.D., Box 3528, Department of Pediatrics, Duke University Medical Center, Durham, NC 27710. Phone: 919-684-2036; FAX: 919-684-8944.

Received for publication 18 September 1995 and accepted in revised form 21 November 1995.

J. Clin. Invest.

(C) The American Society for Clinical Investigation, Inc.

0021-9738/96/02/0941/08 \$2.00

Volume 97, Number 4, February 1996, 941-948

\section{Introduction}

Glycogen storage disease type IV (GSD-IV) ${ }^{1}$ is an autosomal recessive disease caused by deficient glycogen-branching enzyme (GBE) activity. The disease is also referred to as amylopectinosis, because the abnormally stored glycogen has fewer branch points, more $\alpha 1-4$ linked glucose units, and longer outer chains, resulting in a structure resembling amylopectin (1-3). Clinically, GSD-IV is a heterogeneous disorder with remarkable clinical variability. The most common and classical form of GSD-IV is progressive liver cirrhosis, which typically presents in the first 18 mo of life with hepatosplenomegaly and failure to thrive. This is followed by progressive liver cirrhosis with portal hypertension, ascites, esophageal varices, and liver failure, leading to death by $5 \mathrm{yr}$ of age. There are, though less frequently, patients with liver dysfunction who have survived without evidence of progressive liver disease (47). In addition to the hepatic form, a neuromuscular form of the disease has been reported. These patients may $(a)$ present at birth with severe hypotonia, muscle atrophy, and neuronal involvement with death in the neonatal period (8-11); (b) present in late childhood with myopathy or cardiopathy (1214); or $(c)$ present as adults with diffuse central and peripheral nervous system dysfunction accompanied by accumulation of polyglucosan bodies in the nervous system (so-called adult polyglucosan body disease) $(15,16)$.

The molecular basis of GSD-IV is not known, nor is there a known reason for this remarkable clinical variability. All current methods for determination of GBE activity are indirect and therefore not suitable for quantitative analysis of GBE residual activity, and they cannot differentiate the various forms of the disease $(7,8,17)$. At the present time, it is difficult to predict the patient's prognosis after initial diagnosis. It is hoped that the molecular characterization of the different clinical presentations of GSD-IV may yield information that would be helpful in predicting the clinical outcomes, allowing better counseling and treatment.

A human GBE cDNA has been cloned (18). The full length of the cDNA is $\sim 3 \mathrm{~kb}$. The coding sequence contains 2,106 bp encoding a 702-amino acid protein. The calculated molecular mass of the GBE protein, derived from its cDNA sequence, is $80,438 \mathrm{D}$. The human GBE gene has been shown to be located on chromosome 3 (18). The availability of the GBE cDNA will allow study of the disease at the molecular level and unraveling of the molecular mechanisms underlying the clinical variability.

In this paper, we studied the GBE gene in GSD-IV patients with three distinct presentations: the classic presentation of progressive liver cirrhosis and failure; liver disease without

1. Abbreviations used in this paper: GBE, glycogen-branching enzyme; GSD-IV, glycogen storage disease, type IV; RT, reverse transcriptase. 
apparent progression; and the fatal neonatal neuromuscular presentation. We report mutations in the GBE gene in these patients.

\section{Methods}

Patients. Only patients with an enzymatically confirmed diagnosis of GSD-IV were included in this study. All patients were identified through our diagnostic facility for study of glycogen storage disease, except one in whom skin fibroblasts were obtained from National Institute of General Medical Sciences Human Genetic Mutant Cell Repository (Coriell Institute for Medical Research, Camden, NJ; GM 00572). Mutations were studied in detail in four patients with the following clinical histories.

Patient 1 was a neonate who presented at birth with profound hypotonia, muscle atrophy, and dilated cardiomyopathy, and died at 4 wk of age from respiratory and cardiac failure. The clinical and pathologic findings of this infant have been reported (8). This patient represents the neonatal fatal neuromuscular form of the disease.

Patient 2 is a $38 / 12$-yr-old Jewish female who presented at 12 mo of age with failure to thrive, hepatomegaly, and elevation of serum liver transaminases (7). The liver biopsy showed large accumulations of fibrillar periodic acid Schiff-positive material in the hepatocytes, which was partially resistant to diastase digestion. Portal areas appeared broadened with advanced fibrosis. The liver enzymes peaked at the age of $18 \mathrm{mo}$, and then gradually declined and returned to normal by 29 mo of age. During the past 15 mo, her liver function continued to be normal, and there was no clinical evidence of muscle, cardiac, or neurological involvement. This patient represents the nonprogressive hepatic form of the disease.

Patients 3 and 4 presented in early infancy with progressive liver cirrhosis and failure. Patient 4 underwent liver transplantation, and patient 3 died of liver failure before $4 \mathrm{yr}$ of age. These two patients represent the classic hepatic form of the disease.

Total cellular RNA extraction. Total cellular RNA was extracted from cultured skin fibroblasts using acid guanidinium-phenol-chloroform method (19).

Northern blot analysis. Total RNA (10-20 $\mu \mathrm{g})$ was fractionated on a $1 \%$ agarose formaldehyde gel, transferred to a nylon membrane, and hybridized to DNA probes, radiolabeled with $\alpha-\left[{ }^{32} \mathrm{P}\right] \mathrm{dCTP}$ using Megaprime kit (Amersham Corp., Arlington Heights, IL).

Reverse transcriptase (RT)-PCR and subcloning. First-strand cDNAs were synthesized using a gene-specific primer (5'-CTGCATCTGGTGGAGCTGAAATCAG-3' [BE2230(-)], nucleotides 2230-2206 of the published human branching enzyme cDNA sequence), and Superscript RT (GIBCO BRL, Gaithersburg, MD). The second-strand cDNA and additional PCR reactions were carried out using Vent DNA polymerase (New England BioLabs, Inc., Beverly, MA) and a pair of primers that covered the entire coding region of the human GBE gene (nucleotides 88-105, 5'-TTTGGGGGTACCAATATGGCGGCTCCGAT-3' sense [BE88(+)] and 2205-2186, 5'-TTGGGGGAATTCGCCTCTTCAATTCGGCAGAT- ${ }^{\prime}$ antisense [BE2205(-)]). The sense and antisense primers contained additional KpnI and EcoRI linkers, respectively, and, after digestion with KpnI and EcoRI, the amplified cDNA fragments were subcloned into the mammalian expression vector pcDNA3 (Invitrogen Corp., San Diego, CA).

DNA sequencing. Double-stranded dideoxy-chain termination method (20) was performed using $\left[{ }^{35} \mathrm{~S}\right] \mathrm{dATP}$ and Sequenase 2.0 DNA-sequencing kit (United States Biochemical Corp., Cleveland, $\mathrm{OH}$ ) with T7, SP6, or GBE gene-specific primers. Double-stranded DNA cycle sequencing (Perkin-Elmer Corp., Norwalk, CT) was also used for direct sequencing of PCR products.

Screening of genomic DNA library. A genomic DNA library constructed in $\lambda$-GEM 11 (Promega Corp., Madison, WI) was screened by the GBE cDNA probe (21). The probe was labeled with $\alpha-\left[{ }^{32} \mathrm{P}\right] \mathrm{dCTP}$ by the random primer method using the Megaprime kit (Amersham Corp.). The positive clones were partially sequenced by using the cycle sequencing kit (Perkin-Elmer Corp.).

Determination of exon-intron boundary sequences. The exon-intron boundary sequences containing suspected mutations were determined by PCR with the end trimming and cassette ligation method (22). The oligonucleotides used in this procedure are listed in Table I. Briefly, human genomic DNA was digested with three groups of restriction endonucleases: group 1, BamHI, BglII, BstYI, and Sau3AI; group 2, AvrII, NheI, SpeI, and XbaI; group 3, SalI and XhoI. The digests were then ligated with cassettes $\mathrm{MC}$ oligo $+\mathrm{AC} 1$, MC oligo $+\mathrm{AC} 2$, and $\mathrm{MC}$ oligo $+\mathrm{AC} 3$ (Table I) followed by the initial PCRs using MC outer and BE1060(-), or BE950(+) (Table I). These PCR products were further amplified with nested primers $\mathrm{MC}$ inner and BE930(-) or BE1020(+) (Table I). The PCR products were then subcloned into a TA-cloning vector PCR II using TA cloning kit (Invitrogen Corp.). The boundary sequence was determined by the double-stranded dideoxy-chain termination method using Sequenase 2.0.

Verification of mutations in the genomic DNA. To detect the splicing site mutation in patient 1 , genomic DNA was amplified with intronic primers (oligonucleotides 1 and 2, Table II), and the PCR products were sequenced directly by the cycle sequencing method. To confirm further the splicing site mutation, the genomic DNA was amplified with another pair of primers (oligonucleotides 3 and 4, Table II). The resulting PCR products were digested with HincII and electrophoresed on a $12 \%$ polyacrylamide gel (23). To detect mutations R515C and R524X, genomic DNA was amplified with primers (oligonucleotides 5 and 6 , Table II), and the PCR products were digested with Sau3AI or TaqI, respectively. To confirm mutation F257L, genomic DNA was amplified with primers (oligonucleotides 7 and 8 , Table II), and the PCR products were digested with DdeI. To detect L224P and A214T, genomic DNA was amplified with a pair of mismatched primers (oligonucleotides 9 and 10, Table II), and the PCR products were digested with BslI or AluI, respectively. To detect

Table I. Oligonucleotides Used for Determination of Exon-Intron Sequence

\begin{tabular}{|c|c|c|}
\hline Name & Sequences & Notes \\
\hline MC oligo & $\begin{array}{l}\text { 5'CCTCTTCGCTATTACGCCAGTCGACGC- } \\
\text { GGCCGCAAATC3' }\end{array}$ & Main cassette oligomer \\
\hline MC outer & 5'CCTCTTCGCTATTACGCCAG3' & Outer primer annealing to cassette \\
\hline MC inner & 5'AATAAGTCGACGCGGCCGCAAATC3' & Inner primer annealing to cassette \\
\hline AC 1 & 5'ATCGATTTGCGGCCA3' & Adapter cassette oligomer for BamHI, BglII, BstYI, and Sau3AI \\
\hline $\mathrm{AC} 2$ & 5'TAGGATTTGCGGCCA3' & Adapter cassette oligomer for AvrII, NheI, SpeI, and XbaI \\
\hline $\mathrm{AC} 3$ & 5'CGAGATTTGCGGCCA3' & Adapter cassette oligomer for SalI and XhoI \\
\hline BE1060(-) & 5'TATCCCAAAGATCATGAGTCC3' & Antisense outer primer annealing to the human GBE gene \\
\hline BE930(-) & 5'ACCCATGGAATGAGCTGTGT3' & Antisense inner primer annealing to the human GBE gene \\
\hline BE950(+) & 5'TGGTACACAGCCATGCTTCA3' & Sense outer primer annealing to the human GBE gene \\
\hline $\mathrm{BE} 1020(+)$ & 5'TTTTCATTCTGGACCTAGAGGG3' & Sense inner primer annealing to the human GBE gene \\
\hline
\end{tabular}


Table II. Oligonucleotides Used for Genomic DNA Amplifications

\begin{tabular}{|c|c|c|c|}
\hline Oligo No. & Sequence* & Orientation & cDNA coordinates $^{\ddagger}$ \\
\hline 1 & 5'GTGAATTTTGGTTGGGGTAT3' & Sense & Derived from intron ${ }^{\S}$ \\
\hline 2 & 5'ACAAAACAAGGTAAAATCCC $3^{\prime}$ & Antisense & Derived from intron ${ }^{\|}$ \\
\hline 3 & 5'CTTTTTGCTGTGTTCTTCATTG3' & Sense & Derived from intron ${ }^{\S}$ \\
\hline 4 & 5'ACCCATGGAATGAGCTGTGT3' & Antisense & $930-911$ \\
\hline 5 & 5'CAAACATGAGTGTCCTGACTCC3' & Sense & $1592-1613$ \\
\hline 6 & 5'AAGCCCATGCGTAATGAGTC3' & Antisense & $1680-1661$ \\
\hline 7 & 5'TCATGGAGCATGCTTACTAT3' & Sense & $808-828$ \\
\hline 8 & 5'GTCTCTGATTTTACCTGGAA3' & Antisense & Derived from intron ${ }^{\S}$ \\
\hline 9 & 5'TGGGAATTTCTTCCCATGAA3' & Sense & $690-709$ \\
\hline 10 & 5'CCAAGGCCTTTGccTCTTGG3' & Antisense & $782-763$ \\
\hline 11 & 5'TTTTCATTCTGGACCTAGAGGG3' & Sense & $1020-1041$ \\
\hline 12 & 5'TATTGTATGTACCcAtCTGG3' & Antisense & Derived from intron ${ }^{\|}$ \\
\hline
\end{tabular}

* The lowercase letters indicate the mismatched nucleotides. ${ }^{\ddagger}$ Numbered according to published sequences of human glycogen branching enzyme cDNA (18). ${ }^{\S}$ Oligos derived from the intron preceding the 210-bp deletion. " Oligos derived from the intron following the 210-bp deletion.

Y329S mutation, genomic DNA was amplified with primers (oligonucleotides 11 and 12), and the PCR products were digested with BslI.

Construction of GBE mutant cDNAs. To construct the GBE cDNA containing either A214T or L224P, site-directed mutagenesis was performed. The entire coding region of wild-type human brancher cDNA and the cDNA clone that contained both A214T and L224P were used as the templates. The two outside primers were BE88(+) and BE2205( -$)$, which were described above. The two inside primers were BE773(-) 5'-TTGATcCTaGGTAGTACATTGC-3' antisense, which contained two basepair mismatches at nucleotide 765 and 768 to create a restriction enzyme site AvrII, and BE757(+) 5'GTACTACCtAGgATCAAAGGCC-3' sense, which contained two basepair mismatches at nucleotide 765 and 768 to create the same restriction site. These two basepair changes made two silent mutations, which did not alter the coding amino acids. Another inside primer that contained the mutation L224P and the two silent mutations was BE773(-) mutant 5'-TTGATcCTaGGTgGTACATTGC-3' antisense. The mismatched and mutant bases are indicated by lowercase letters. PCRs with two pairs of primers, BE88(+) and BE773(-), $\mathrm{BE757}(+)$ and BE2205(-), were performed on both the wild-type and the cDNA clone which contained A214T and L224P, to create the wild-type with the silent mutations and the A214T with the silent mutations. PCRs with two pairs of primers, BE88(+) and BE773(-) mutant, BE757(+) and BE2205(-), were performed on the wild-type cDNA clone to create the L224P with the silent mutations. The PCR products were digested with KpnI, AvrII, and EcoRI and then ligated into pcDNA3 vector. All constructs were confirmed by DNA sequencing. Other mutant clones containing either R515C, F257L, R524X, or Y329S were obtained directly from the original RT-PCR products.

Transient transfection in COS-7 cells. To confirm the mutations causing GSD-IV, transient expression experiments were performed in COS-7 cells. The wild-type or mutant cDNA in a pcDNA3 vector (Invitrogen Corp.) was transfected into COS-7 cells by the DEAEdextran method (24). Mock transfections of COS-7 cultures with pcDNA3 vector were used as controls. After incubation at $37^{\circ} \mathrm{C}$ for $24 \mathrm{~h}$, the transfected cultures were subcultured (1:2 split) and incubated for an additional $24 \mathrm{~h}$. One culture was harvested for branching enzyme assays, and in some cases the other culture was used for RNA isolation. For each cDNA clone, four transfection experiments were performed, and the results were expressed as mean and range of four separate determinations.

Enzyme assay for branching enzyme activity. Branching enzyme activity was determined using phosphorylase as the indicating enzyme $(3,6)$.

\section{Results}

Northern blot analysis of GBE in patients and normal controls

The branching enzyme mRNA in normal control is $\sim 3 \mathrm{~kb}$ (Fig. 1, lane 1). The neonate with fatal neuromuscular presentation of GSD-IV demonstrated a smaller size and reduced amount of GBE mRNA (lane 2). Three patients who presented with progressive liver cirrhosis and failure, followed by either liver transplantation or death in early childhood, were shown to have either a reduced amount (lanes 4 and 5) or a trace amount (lane 3 ) of brancher mRNA. Two other patients (lanes 6 and 7) who presented with liver disease but no progression had normal amounts and sizes of brancher mRNA. No striking difference in the $\gamma$-actin mRNA levels was observed among these samples. Southern blot analysis of genomic DNA digested with EcoRI, HindIII, or MspI did not detect gross DNA rearrangements, deletions, or insertions in the GBE gene of these patients (data not shown).

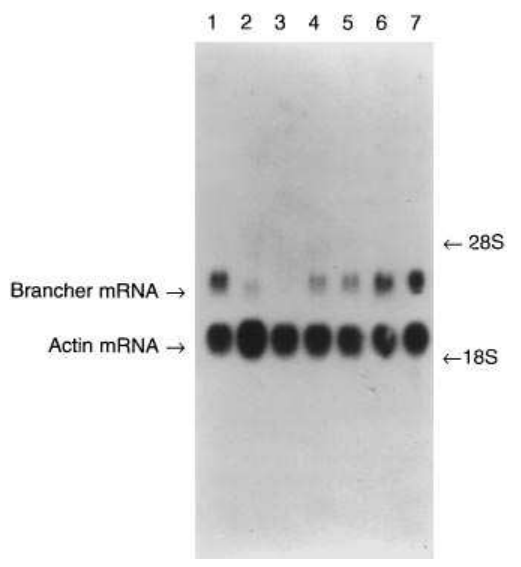

Figure 1. Northern blot analysis of branching enzyme mRNA in patients and normal controls. $20 \mu \mathrm{g}$ total cellular RNA extracted from the skin fibroblasts of normal control and GSD-IV patients was loaded on a $1 \%$ denatured formaldehyde agarose gel. The fractionated RNAs were then transferred to a nylon membrane and hybridized with a GBE cDNA probe that was labeled with $\alpha-\left[{ }^{32} \mathrm{P}\right] \mathrm{dCTP}$ by random primer method. The membrane was then hybridized with $\gamma$-actin probe as the internal control. The blot represents a single exposure of two probes hybridized sequentially. Lane 1, Normal control. Lane 2, patient with the neonatal neuromuscular form. Lanes $3-5$, patients with progressive liver cirrhosis and failure. Lanes 6 and 7 , patients with the nonprogressive hepatic form. 

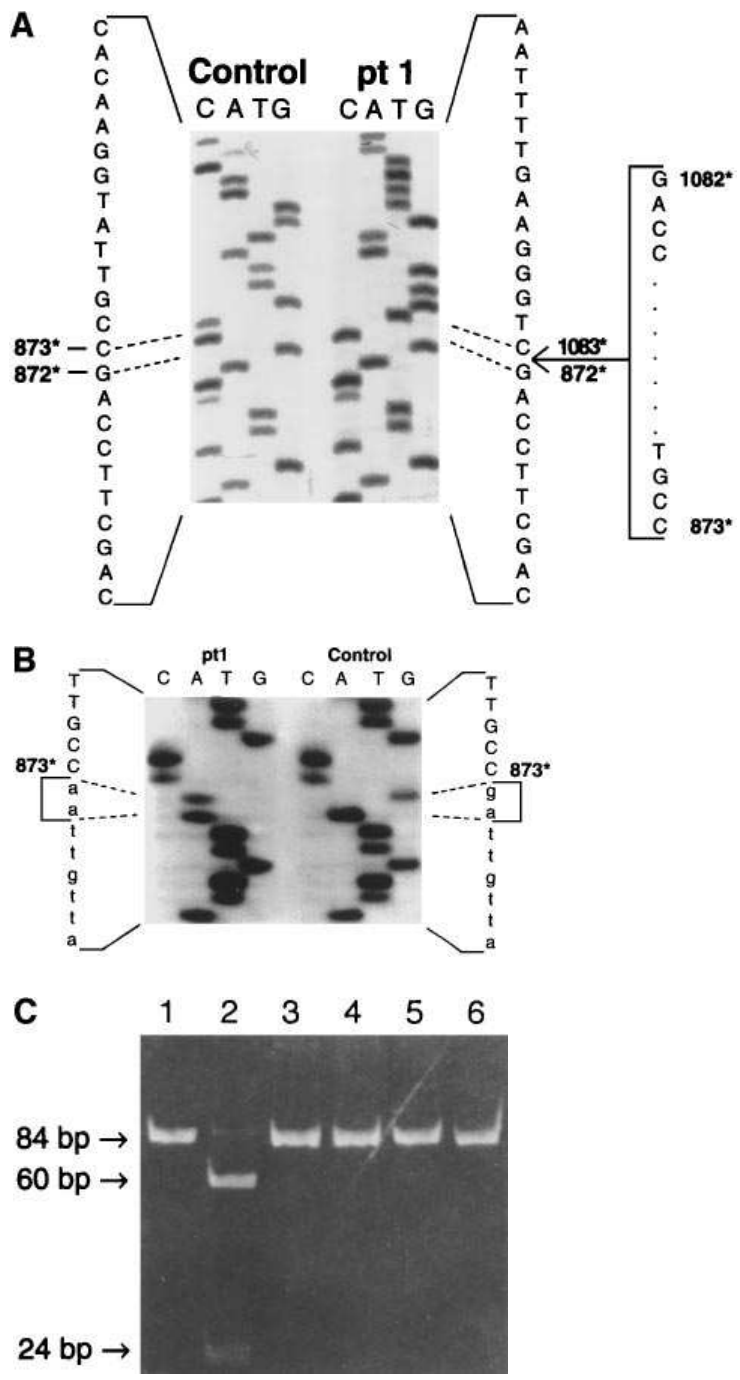

Figure 2. Mutation study in patient 1. (A) A 210-bp deletion from cDNA nucleotide 873 to 1082 that caused 70 amino acids missing from GBE polypeptide. (B) Partial sequences of PCR-amplified genomic products showing a $g$-to- $a$ substitution at the $3^{\prime}$ end of an intron. Lowercase letters indicated the intron sequence. $(C)$ HincII digestion of PCR-amplified genomic DNA fragments. Digestion of mutant DNA yielded two fragments (60 and $24 \mathrm{bp}$ ), whereas normal allele remained intact as $84 \mathrm{bp}$. Lane 1, normal control. Lane 2, patient 1. Lanes 3-5, GSD-IV patients with progressive liver cirrhosis.

\section{Identification and characterization of mutations}

A 3' acceptor splicing site mutation was present in a patient with neonatal fatal neuromuscular form of the GSD-IV. A 210-bp deletion from nucleotide 873 to 1082 was detected in all 10 cDNA clones obtained from subcloning of the RT-PCR products from patient 1 (Fig. $2 A$ ), consistent with the smaller size of brancher mRNA in the Northern blot (Fig. 1, lane 2). This deletion resulted in a loss of 70 amino acids from the GBE polypeptide (262-331). The deletion did not shift the reading frame and did not change any of the remaining amino acid sequence. Southern blot analysis of genomic DNA using the 210bp region that was absent from mRNA as a probe showed a normal pattern (data not shown). The $210 \mathrm{bp}$, therefore, were deleted during RNA processing, most likely because of a splicing junction mutation. Using PCR with end trimming and cas- sette ligation method (22), the $210 \mathrm{bp}$ were shown to be a single exon. The 5' and $3^{\prime}$ boundary sequences of this exon were determined and used as primers (Table II, oligo 1 and 2) for PCR. Direct cycle sequencing of the PCR products showed a 3 'acceptor splicing site mutation changing $a g$ to $a a$. The mutation created a new restriction enzyme site for HincII that was not present in the normal allele. PCR amplification of genomic DNA showed an 84-bp fragment in normal control. After HincII digestion, the 84-bp fragment remained intact in normal control. However, a 60-bp and a 24-bp fragment were observed in patient 1 (Fig. $2 C$ ). The result is consistent with homozygosity or heterozygosity with failure of the other chromosome to amplify in the PCR analysis. The completely abolished branching enzyme activity by this deletion was demonstrated by the transient transfection experiments in COS-7 cells (Table III).

Point mutations were detected in a patient with nonprogressive hepatic form of GSD-IV. $13 \mathrm{cDNA}$ clones were sequenced from patient 2 (Fig. 1, lane 6), who had a nonprogressive hepatic form of the disease. A G-to-A transition at nucleotide 730 and a T-to-C transition at nucleotide 761 (Fig. 3 A) were detected in four clones. The other eight clones contained an A-to-C transversion at nucleotide 1076 (Fig. 3 B). The G-to-A transition at nucleotide 730 caused an $\mathrm{Ala}^{214}$-to-Thr substitution (A214T) and the T-to-C transition at 761 caused a Leu ${ }^{224}$ to-Pro substitution (L224P). The $\mathrm{A}^{1076}$-to-C transversion caused a $\mathrm{Tyr}^{329}$-to-Ser substitution (Y329S).

To ensure that the point mutations detected in cDNA clones were not PCR artifacts, genomic DNA was studied. We first determined the exon-intron boundary sequences around these mutations and used this information to design primers for PCR. The A214T and L224P were located in a single exon, and Y329S was 4 bp away from the $3^{\prime}$ border of another exon. By restriction analysis of PCR-amplified genomic products, these mutations were confirmed. Family study showed that the proband inherited L224P from her father, and neither her mother nor two unaffected siblings had the allele (Fig. $3 \mathrm{C}$ ). The A214T allele also followed the same inheritance pattern

Table III. Branching Enzyme Activities of Transfected COS-7 Cells in Transient Expression Experiments

\begin{tabular}{ll} 
Constructs & Branching enzyme activity \\
\hline nmoles/min per $m$ m protein
\end{tabular}

Mock*

Wild type

$523(390-890)^{\ddagger}$

Wild type with silent mutations ${ }^{\S}$

6046 (5478-6699)

210 bp deletion mutant allele

6457 (5735-7893)

A214T with silent mutations $\$$

487 (363-557)

L224P with silent mutations ${ }^{\S}$

6073 (5971-6210)

Y329S mutant allele

$529(341-849)$

R515C mutant allele

3250 (2545-3685)

F257L mutant allele

1194 (863-1488)

R524X muant allele

1649 (1478-1846)

$535(257-704)$

* COS-7 cells transfected with pcDNA vector alone. ${ }^{\ddagger}$ Values represent mean and ranges of four separate determinations with ranges in parentheses. ${ }^{\S}$ Silent mutations at nucleotide $A^{765}$ to $T$ and $A^{768}$ to $G$ creating an AvrII site for site-directed mutagenesis. See text for details. 
A

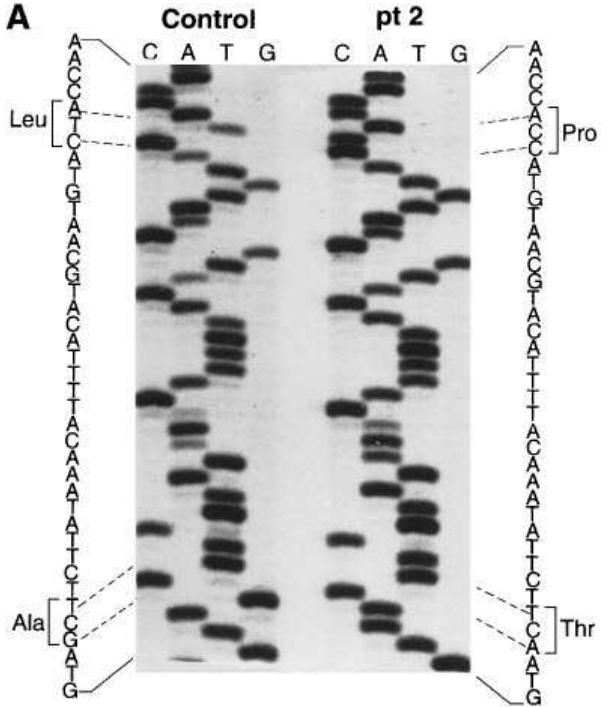

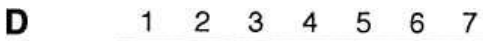

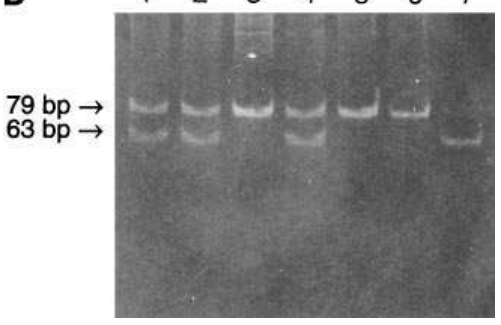

E

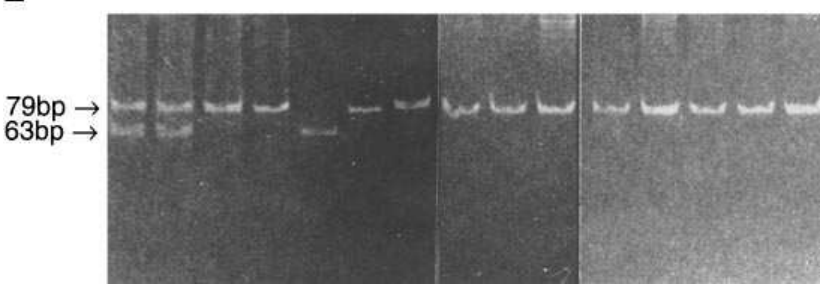

B
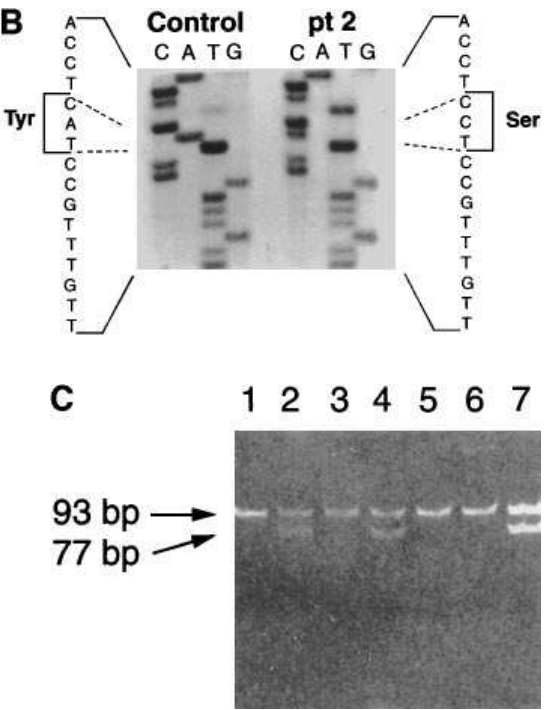

Figure 3. Mutation study in patient 2. (A) Partial sequencing of cDNA clones. The GBE gene of patient 2 contained a G-to-A transition at nucleotide 730 and a T-to-C transition at nucleotide 761, which caused an $\mathrm{Ala}^{214}$-to-Thr substitution (A214T) and Leu $^{224}$-to-Pro substitution (L224P), respectively. (B) Partial sequencing data of cDNA clones. The other allele of GBE gene contained an A-to-C transversion at nucleotide 1076, which caused a $\mathrm{Tyr}^{329}$ to-Ser substitution (Y329S). (C) BslI digestion of PCR-amplified genomic DNA fragments. Digestion of mutant DNA yielded two fragments (77 and $16 \mathrm{bp}$; the 16-bp fragment was too small to be seen), whereas normal DNA remained intact as $93 \mathrm{bp}$. Lane 1, normal individual. Lanes 2-6 were from proband, mother, father, and unaffected siblings 1 and 2, respectively. Lane 7, mixtures of mutant and normal cDNA clones as an internal control. (D) Family study of Y329S allele. BsII digestion of PCR-amplified genomic DNA fragments. Digestion of mutant DNA yielded two fragments (63 and 16 bp; the 16-bp fragment was too small to be seen), whereas normal allele remained intact as $79 \mathrm{bp}$. Lanes $1-5$ were proband, mother, father, and unaffected siblings 1 and 2, respectively. Lane 6, normal individual. Lane 7, Y329S cDNA clone. $(E)$ Y329S allele in other patients and controls. The same restriction analysis of PCR-amplified genomic products as Fig. 3 $D$ was used. Lane 1 , patient 2. Lanes 2-4, other patients with nonprogressive hepatic GSD-IV. Lane 5, Y329S cDNA clone as an internal control. Lanes 6-11, patients with progressive liver cirrhosis and failure. Lanes 12 and 13, patients with fatal neuromuscular GSD-IV. Lanes 14 and 15 , normal individuals.

as L224P (data not shown), indicating that these two alleles are on the same chromosome, consistent with the cDNA data. The proband's other allele, Y329S, was inherited from her mother, and one of the two unaffected siblings also had the mutant allele, suggesting carrier status (Fig. $3 \mathrm{D}$ ).

Transient transfection experiments were performed to determine which mutations resulted in disease phenotype. Since A214T and L224P appeared on the same chromosome, sitedirected mutagenesis was performed to separate these two mutations into two cDNA clones that contained either A214T or L224P. To make GBE cDNA containing either A214T or L224P, a restriction enzyme site (AvrII) was created by introducing two silent mutations ( $A^{765}$ to $T$ and $A^{768}$ to $G$ ) for the purpose of ligation and subcloning. Transfection of cDNA containing the L224P allele resulted in markedly reduced GBE activity compared with experiments with wild-type cDNA and wild-type cDNA with silent mutations (Table III). The cDNA containing A214T allele, in contrast, had normal GBE activity (Table III). This indicated that the L224P mutation, but not A214T, is the disease-causing mutation. The Y329S allele, however, had $\sim 50 \%$ of GBE activity compared with the wild type (Table III). Northern blot analysis of branching enzyme transcripts from transfected COS-7 cells showed that the wild-type and mutant brancher mRNAs were expressed at similar levels (data not shown), indicating that the degree of reduction of branching enzyme activity among the mutant alleles compared with the wild-type allele was unlikely to have been caused by differences in the efficiency of transfection and/or transcription.

Y329S was detected only in patients with nonprogressive hepatic form of GSD-IV. To test whether Y329S represents a common polymorphism, we screened 35 unrelated control individuals. The Y329S mutation was not present in any of these control individuals. We also screened for the Y329S mutation in 11 additional GSD-IV patients ( 6 with progressive liver cirrhosis and failure, 2 with fatal neonatal neuromuscular presentation, and 3 with the nonprogressive hepatic form). The Y329S mutation was not detected in any of the more severe forms of GSD-IV (Fig. $3 \mathrm{E}$ ). The Y329S allele was, however, detected in another GSD-IV patient who had liver disease without progression (Fig. $3 \mathrm{E}$ ). This patient is currently $20 \mathrm{yr}$ old and has normal liver function (7). 


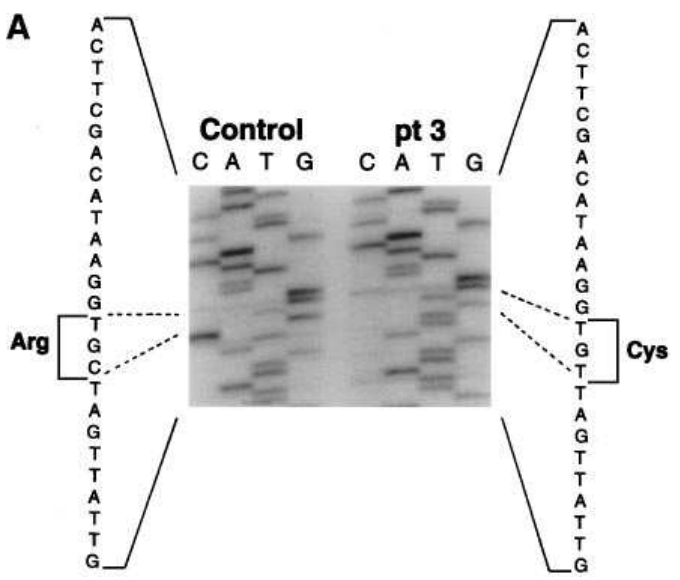

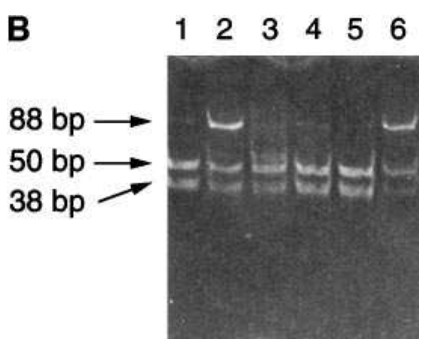

Figure 4. Mutation study in patient 3. (A) Partial DNA sequences of direct sequencing of RT-PCR products showing a C-to- $\mathrm{T}$ transition at nucleotide 1633 that changed an $\mathrm{Arg}^{515}$ to Cys (R515C). (B) Sau3AI digestion of PCRamplified genomic DNA fragments. Digestion of normal DNA yielded two fragments (50 and $38 \mathrm{bp}$ ), whereas mutant DNA remained intact as 88 bp. Lane 1, normal individual. Lane 2, patient 3. Lanes 3-5, patients 1,2 , and 4, respectively. Lane 6 , mixture of normal and mutant cDNA clones as an internal control.
Point mutations detected in patients with classical presentation of GSD-IV. Three point mutations were found in patients 3 and 4, who had the classical clinical presentation of progressive liver cirrhosis and failure. A point mutation that involved a C-to-T transition at cDNA sequence 1633 that changed $\mathrm{Arg}^{515}$ to Cys (R515C) was detected in 19 of 20 cDNA clones from patient 3 . This mutation was also detected by direct sequencing of the RT-PCR product (a single band as shown in Fig. $4 A$ ). The mutation caused a loss of the restriction enzyme site Sau3AI. Using a pair of primers (Table II, oligonucleotides 5 and 6), genomic DNA was amplified and digested with Sau3AI. An 88-bp fragment amplified from patient 3 was digested into three bands of 88,50 , and 38 bp (Fig. $4 \mathrm{~B}$ ). This suggested that patient 3 was a compound heterozygote with another allele, presumably mRNA negative (decreased transcription or unstable mRNA), consistent with the decreased amount of GBE mRNA in the Northern blot (Fig. 1, lane 4).
In patient 4 , nine cDNA clones obtained by subcloning of the RT-PCR products were sequenced. Seven clones carried a T-to-A transversion at cDNA position 861 that changed $\mathrm{Phe}^{257}$ to Leu (F257L) (Fig. $5 \mathrm{~A}$ ), and two clones contained a C-to-T transition at nucleotide 1660 that altered $\mathrm{Arg}^{524}$ to a stop codon (R524X) (Fig. 5 B). To confirm the mutation in the patient's genomic DNA, the exon-intron junction sequence was determined by direct sequencing of the genomic clones containing the mutation. Since F257L is close to the $3^{\prime}$ end of an exon, the genomic DNA was amplified with two primers: The sense strand primer was generated from the exon and the antisense strand primer from the intron (Table II, oligonucleotides 7 and 8). A 78-bp fragment was amplified. The mutation created a new DdeI site. The restriction pattern of the digest showing three fragments suggested that patient 4 is heterozygous for the F257L mutation (Fig. 5 C). For mutation R524X, an 88-bp fragment was amplified from genomic DNA using

A
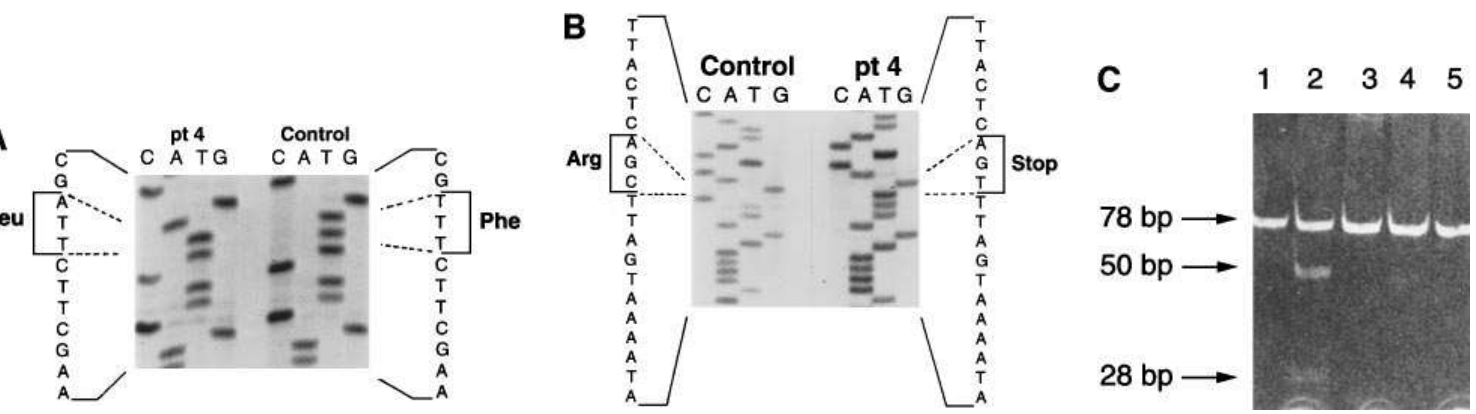

D $\begin{array}{llllll}1 & 2 & 3 & 4 & 5 & 6\end{array}$

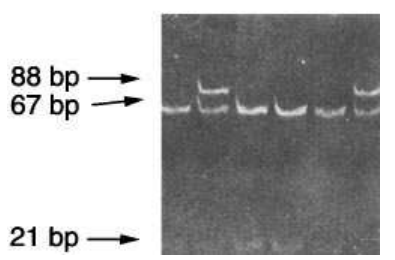

Figure 5. Mutation study in patient 4. (A) Partial sequencing of cDNA clones. The GBE gene of patient 4 contained a T-to-A transversion at nucleotide 861, which changed a Phe ${ }^{257}$ to Leu (F257L). (B) Partial sequencing of cDNA clones. The other allele of the GBE gene of patient 4 contained a C-to-T transition at nucleotide 1660, which altered an $\mathrm{Arg}^{524}$ to a stop codon (R524X). (C) DdeI digestion of PCR-amplified genomic DNA fragments. Digestion of mutant DNA yielded two fragments (50 and $28 \mathrm{bp}$ ), whereas normal DNA remained intact as $78 \mathrm{bp}$. Lane 1, normal individual. Lane 2, patient 4. Lanes 3-5, patients 1, 2, and 3, respectively. (D) TaqI digestion of PCR-amplified genomic DNA fragments. Digestion of normal DNA yielded two fragments (67 and $21 \mathrm{bp}$ ), whereas mutant DNA remained intact as $88 \mathrm{bp}$. Lane 1, normal control. Lane 2 , patient 4. Lanes 3-5, patients 1, 2, and 3, respectively. Lane 6, mixture of mutant and normal cDNA clones. 
primers 5 and 6 (Table II). The mutation eliminated a TaqI site. The restriction pattern of the digest (three bands at 88, 67, and $21 \mathrm{bp}$ ) confirmed that the other allele of patient 4 carried the mutation R524X (Fig. $5 \mathrm{D}$ ).

None of the three point mutations in patients 3 and 4 were present in normal controls or eight other GSD-IV patients studied. These mutations inactivated the GBE activities to various levels, ranging from 80 to $99 \%$ (Table III).

Other sequence variations. A deviation of the published GBE cDNA sequences (18) was observed at nucleotide 353 involving a C-to-G change. This nucleotide difference, which altered amino acid 88 from serine to cysteine, was detected in all cDNA clones from controls and patients. We believe this represents the correct sequence and that the sequence in early reports represents either a rare variant or an artifact. An A-to-G transition at nucleotide 658 that altered $\operatorname{Arg}^{190}$ to Gly was observed both in controls and in patients, with 67 and $33 \%$ frequencies of $\mathrm{A}$ and $\mathrm{G}$ alleles, respectively. This nucleotide change appeared to be a polymorphism as frequencies of the two alleles in GSD-IV patients were not different from that in the controls.

\section{Discussion}

Although progressive liver cirrhosis is the classic and most common clinical presentation for GSD-IV, a review of the literature (1-16) and the patients presented here demonstrate significant clinical variability. Enzymatically, except for adult polyglucosan body disease $(15,16)$, most GSD-IV patients have what appears to be a generalized enzyme defect. The deficiency of GBE activity and the accumulation of amylopectin can be demonstrated in almost all tissues studied, including liver, heart, skeletal muscle, brain, leukocytes, and fibroblasts $(5,9,12)$. The differing degree of clinical severity and organ involvement is unlikely to be due to defects in tissue-specific isoenzymes encoded by different genes expressed in different tissues, since there is no clear evidence for the existence of isoforms of the branching enzyme $(25,26)$. Based on these data, we hypothesized that the various forms of GSD-IV are encoded by mutations in the same GBE structural gene. GSD-IV patients studied in this work represented three very different clinical presentations: neonates with a fatal neuromuscular disorder without liver fibrosis, patients with progressive liver cirrhosis and failure, and patients with liver disease but without apparent progression. Despite the remarkable clinical variability, we have identified molecular defects in the same GBE gene, albeit different mutations, in these three different forms of GSD-IV. Thus, these various forms of GSD-IV represented an allelic heterogeneity of the GBE gene.

When an infant presenting with liver cirrhosis is diagnosed as GSD-IV, the decision for liver transplantation is complicated by the difficulty in predicting how the disease will progress: either liver failure and death, or a benign course (4-7). Our finding of a significant residual GBE activity (50\% control) in the mutant Y329S allele may have clinical implications. This mutant allele was found in two out of four nonprogressive hepatic GSD-IV patients, but in none of the more severe GSD-IV patients (two with the fatal neonatal neuromuscular presentation and six with the progressive liver cirrhosis and failure). The Y328S allele also was not present in any of the 35 unrelated control individuals. The presence of significant GBE activity in the Y329S allele might be a reason why patients with the mild hepatic form of the disease are protected from progression to liver failure.

It is interesting to note that, except for the deletion, none of the missense mutations involved proposed catalytically active sites of the GBE. The 210-bp deletion caused by the $3^{\prime}$ acceptor splicing site mutation in patient 1 is located in a region that is highly conserved between prokaryotic, yeast, and human branching enzyme polypeptide sequences (27), and also includes the first of the four regions (DVVHS) that constitute the catalytically active sites of most amylolytic enzymes $(27,28)$. The deletion completely abolished GBE activity in transient expression experiments. The missense mutations identified in this study also diminished the GBE activity; however, how these point mutations affect the GBE activity is not clear. This will await structure-function analysis of the mutant proteins.

We conclude that the three distinct forms of GSD-IV all result from mutations in the same GBE gene. Further study of genotype/phenotype correlation may yield information useful in predicting the clinical outcomes.

\section{Acknowledgments}

We thank Dr. Thomas T. Tang (Children's Hospital of Wisconsin), Dr. Arthur L. Beaudet (Baylor College of Medicine), and Dr. J. Timothy Boyle (Rainbow Babies and Children's Hospital) for providing patient samples, and Ms. Quinn Sadler for her expert secretarial assistance.

This work was supported in part by grants DK 39078 and M01RR30 (General Clinical Research Centers Program, National Center for Research Resources, National Institutes of Health) and by the glycogen storage disease IV fund (Duke University).

\section{References}

1. Chen, Y.-T., and A. Burchell. 1995. Glycogen storage diseases. In The Metabolic and Molecular Bases of Inherited Diseases. 7th ed. C.R. Scriver, A.L. Beaudet, W.S. Sly, and D. Valle, editors. McGraw-Hill, New York. 935965.

2. Servidei, S., and S. DiMauro. 1989. Disorders of glycogen metabolism of muscle. Neurologic Clinics. 7:159-178.

3. Brown, B.I., and D.H. Brown. 1966. Lack of an $\alpha-1,4$ glucan: $\alpha-1,4$ glucan 6-glycosyl transferase in a case of type IV glycogenosis. Proc. Natl. Acad. Sci. USA. 56:725-729.

4. Guerra, A.S., O.P. van Diggelen, F. Carneiro, R.M. Tsou, S. Simoes, and N.T. Santos. 1986. A juvenile variant of glycogenosis IV (Andersen disease). Eur. J. Pediatr. 145:179-181.

5. Greene, H.L., B.I. Brown, D.T. McClenahan, R.M. Agostini, and S.R. Taylor. 1988. A new variant of type IV glycogenosis: deficiency of branching enzyme activity without apparent progressive liver disease. Hepatology. 8:302306.

6. Brown, B.I., and D.H. Brown. 1989. Branching enzyme activity of cultured amniocytes and chorionic villi: prenatal testing for type IV glycogen storage disease. Am. J. Hum. Genet. 44:378-381.

7. McConkie-Rosell, A., C. Wilson, D.A. Piccoli, J. Boyle, T. DeClue, P. Kishnani, J.-J. Shen, A. Boney, B. Brown, and Y.-T. Chen. 1996. Clinical and laboratory findings in four patients with a nonprogressive hepatic form of type IV glycogen storage disease. J. Inherited Metab. Dis. In press.

8. Tang, T.T., A.D. Segura, Y.-T. Chen, L.M. Ricci, R.A. Franciosi, M.L. Splaingard, and M.S. Lubinsky. 1994. Neonatal hypotonia and cardiomyopathy secondary to type IV glycogenosis. Acta Neuropathol. 87:531-536.

9. van Noort, G., W. Straks, O.P. Van Diggelen, and R.C.M. Hennekam. 1993. A congenital variant of glycogenosis type IV. Pediatr. Pathol. 13:685-698.

10. Herrick, M.K., J.L. Twiss, G.D. Vladutiu, G.F. Glassocck, and D.S Horoupian. 1994. Concomitant branching enzyme and phosphorylase deficiencies. An unusual glycogenosis with extensive neuronal polyglucosan storage. $J$. Neuropathol. \& Exp. Neurol. 53:239-246.

11. Zellweger, H., S. Mueller, V. Ionasescu, S.S. Schochet, and W.F. McCormick. 1972. Glycogenosis IV: a new cause of infantile hypotonia. J. Pediatr. 80:842-844.

12. Servidei, S., R.E. Riepe, C. Langston, L. Tani, J.T. Bricker, N. Crisp- 
Lindgren, H. Travers, D. Armstrong, and S. DiMauro. 1987. Severe cardiopathy in branching enzyme deficiency. J. Pediatr. 111:51-56.

13. Reusche, E., F. Aksu, H.H. Goebel, Y.S. Shin, T. Yokota, and H. Reichmann. 1992. A mild juvenile variant of type IV glycogenosis. Brain \& Dev. 14: $36-43$

14. Schroder, J.M., R. May, Y.S. Shin, M. Sigmund, and S. Nase-Huppmeier. 1993. Juvenile hereditary polyglucosan body disease with complete branching enzyme deficiency (type IV glycogenosis). Acta Neuropathol. 85: $419-430$.

15. Lossos, A., V. Barash, D. Soffer, Z. Argov, M. Gomori, Z. Ben-Nariah, O. Abramsky, and I. Steiner. 1991. Hereditary branching enzyme dysfunction in adult polyglucosan body disease: A possible metabolic cause in two patients. Ann. Neurol. 30:655-662.

16. Bruno, C., S. Servidei, S. Shanske, G. Karpati, S. Carpenter, D. McKee, R.J. Barohn, M. Hirano, Z. Rifai, and S. DiMauro. 1993. Glycogen branching enzyme deficiency in adult polyglucosan body disease. Ann. Neurol. 33:88-93.

17. Brown, D.H., and B.I. Brown. 1983. Studies of the residual glycogen branching enzyme activity present in human skin fibroblasts from patients with type IV glycogen storage disease. Biochem. Biophys. Res. Commun. 111:636642

18. Thon, V.J., M. Khalil, and J.F. Cannon. 1993. Isolation of human glycogen branching enzyme cDNAs by screening complementation in yeast. J. Biol. Chem. 268:7509-7513.

19. Chomczynski, P., and N. Sacchi. 1987. Single-step method of RNA isolation by acid guanidinium thiocyanate-phenol-chloroform extraction. Anal. Biochem. 162:156-159.

20. Sanger, F., S. Nicklen, and A.R. Coulson. 1977. DNA sequencing with chain-termination inhibitors. Proc. Natl. Acad. Sci. USA. 74:5463-5467.

21. Benton, W.D., and R.W. Davis. 1977. Screening $\lambda$ gt recombinant clones by hybridization to single plaques in situ. Science (Wash. DC). 196:180-182.

22. Iwahana, H., T. Tsujisawa, R. Katashima, K. Yoshimoto, and M. Itakura. 1994. PCR with end trimming and cassette ligation: a rapid method to clone exon-intron boundaries and a $5^{\prime}$-upstream sequence of genomic DNA based on a cDNA sequence. PCR Methods Applic. 4:19-25.

23. Ding, J.-H., B.-Z. Yang, Y. Bao, C.R. Roe, and Y.-T. Chen. 1992. Identification of a new mutation in medium-chain acylCoA dehydrogenase deficiency. Am. J. Hum. Genet. 50:229-233.

24. Aruffo, A. 1991. Transient expression of proteins using COS cells. In Current Protocols in Molecular Biology. F.M. Ausubel, R. Brent, R.E. Kingston, D.D. Moore, J.G. Seidman, J.A. Smith, and K. Struhl, editors. Greene Publishing and Wiley-Interscience, New York. 16.13.1-16.13.7.

25. Zimmerman, C.P., and A.M. Gold. 1983. Isolation and characterization of glycogen branching enzyme from rabbit liver. Biochemistry. 22:3387-3392.

26. Satoh, K., and K. Sato. 1991. Purification of RNA associated and unassociated forms of 1.4- $\alpha$-glucan branching enzyme from rat liver. J. Biochem. 91: 1129-1137.

27. Thon, V.J., C. Vigneron-Lesens, T. Marianne-Pepin, J. Montreuil, A. Decq, C. Rachez, S.G. Ball, and J.F. Cannon. 1992. Coordinate regulation of glycogen metabolism in the yeast Saccharomyces cerevisiae. J. Biol. Chem. 267 $15224-15228$.

28. Baba, T., K. Kimura, K. Mizuno, H. Etoh, Y. Ishida, O. Shida, and Y. Arai. 1991. Sequence conservation of the catalytic regions of amylolytic enzymes in maize branching enzyme-I. Biochem. Biophys. Res. Commun. 181:87- 